\title{
Potential role of heat-shock proteins in giant cell tumors
}

\author{
J.T. Chen, A. Younusi, L. Cao, Z. Tian, Y.J. Zhou and X.H. Song \\ Orthopaedic Department, \\ The First Affiliated Hospital of XinJiang Medical University, Urumqi, China \\ Corresponding authors: X.H. Song / J.T. Chen \\ E-mail: gentelchan@163.com / gentelchan@126.com
}

Genet. Mol. Res. 14 (4): 19144-19154 (2015)

Received August 14, 2015

Accepted October 25, 2015

Published December 29, 2015

DOI http://dx.doi.org/10.4238/2015.December.29.24

ABSTRACT. We investigated the differential expression protein profile of giant cell tumors (GCTs), which can be used to monitor the tumor's recurrence and metastasis, to provide preliminary results for further study. We also explored heat-shock protein (HSP) inhibitor that prevents tumors from recurring and migrating. A stable isotope-labeling strategy using isobaric tags for relative and absolute quantitation coupled with twodimensional liquid chromatography tandem mass spectrometry was used to separate and identify differentially expressed proteins. A total of 467 differentially expressed proteins were identified in GCT tissues. Up to 311 proteins were upregulated, whereas 156 proteins were downregulated in GCT tissues. Three of the differentially expressed HSPs, namely HP90A, HSPB1, and HSPB2, were upregulated. The differentially expressed proteins of GCT tissues will provide a scientific foundation for tumor prognosis, and for further studies exploring HSP inhibitor to prevent tumor recurrence and migration.

Key words: Heat-shock proteins; Giant cell tumor; iTRAQ; 2-D LC-MS/MS 


\section{INTRODUCTION}

Giant cell tumor (GCT) is a bone-destructive neoplasm with a generally benign course, and a benign bone tumor with fairly high local aggressiveness (Gupta et al., 2008). Development of lung metastases have been observed in rare cases, i.e., 2-5\% (Campanacci, 1993; Gamberi et al., 1998). Histologically, the tumor pattern is formed by a network of multinucleated osteoclastlike giant cells, spindle-shaped fibroblast-like stromal cells, round mononuclear histiocytic cells, and CD68-positive phagocytic histiocytes (Werner, 2006). A few proteomics analysis studies have been reported (Conti et al., 2011; Zhang et al., 2013). However, the use of isobaric tags for relative and absolute quantitation (iTRAQ) technology to identify the differentially expressed proteins associated with GCT has not been reported to date.

Heat-shock proteins (HSPs) have important roles in the carcinogenesis of some tumors. HSPs represent a group of novel molecular biomarkers in cancer management. HSPs are a large class of proteins that have been highly conserved throughout evolution and are expressed by prokaryotic and eukaryotic organisms. HSPs are usually divided into seven family members based on their molecular size (Badowska-Kozakiewicz and Malicka, 2012). Proteomic and genomic studies have shown that HSPs may be linked to an increased risk of developing ovarian cancer (Chow et al., 2010). The serum HSP27 levels are a potential additional indicator of peritoneal metastases in epithelial ovarian cancer, and may be used to monitor treatment response (Zhao et al., 2014). HSP90 inhibits apoptosis in cancer cells, and enhances angiogenesis and cell cycle progression. A possible mechanism of the amniotic membrane anti-cancer effect is through inhibition of HSP90 (Niknejad et al., 2013). Other studies have also shown the essential role of HSPs in malignant processes (Cohen et al., 2010). This study sought to recognize and identify differentially expressed proteins, especially the HSPs, and pathways associated with GCT. Furthermore, we identified the relationship between GCT and HSPs, including the recurrence and metastasis of the tumor, and explored HSP inhibitor to prevent tumor recurrence and migration. The results will help clinicians make prognoses and select appropriate therapies. For this purpose, we used iTRAQ, a novel proteomic method. Aside from two-dimensional (2-D) differential in-gel electrophoresis or proteomic methods based on stable isotope labeling (e.g., isotope-coded affinity tag), the ITRAQ technique is very suitable, especially for comparative studies involving eight samples (the samples should be evaluated in parallel) (Ross et al., 2004; Yan and Chen, 2005). This approach is more sensitive than 2-D differential in-gel electrophoresis and isotope-coded affinity tag (Wu et al., 2006). In contrast to methods that use stable isotope labeling, iTRAQ enables all samples to be processed simultaneously, thereby reducing analysis time (Shadforth et al., 2005). The major advantage of iTRAQ over 2-D electrophoresis, which is the most commonly used method in proteomics, lies in the possibility of being able to detect lowlevel expression of proteins and integral membrane proteins (Ernoult et al., 2008). Membrane proteins must first be solubilized by detergents before 2-D electrophoresis, which can be difficult (Molloy, 2000; Rabilloud, 2009). iTRAQ is a powerful proteomic approach based on the use of four amine-specific isobaric reagents that label the primary amines of peptides from four different biological samples. These isobaric mass labels are attached to the $\mathrm{N}$-termini and lysine side chains of peptides, and produce abundant tandem mass spectrometry (MS/MS) signature ions. Their relative peak areas are determined by the relative proportions of the labeled peptides $(\mathrm{Wu}$ et al., 2006). 


\section{MATERIAL AND METHODS}

\section{Ethics statement}

The research protocol was approved by the Ethics Committee of The First Affiliated Hospital of XinJiang Medical University (protocol No. 20130216-09). All the patients provided appropriate informed consent. All diagnoses were confirmed by pathologists. The four patients selected had complete medical records. The GCT and normal bone tissues were collected at the same time. Normal bone tissues were used for comparison, and were obtained sufficiently far from the tumor tissues $(5 \mathrm{~cm})$ in the same limb.

\section{Protein preparation}

GCT samples were ground into powder in liquid nitrogen, extracted with lysis buffer containing $1 \mathrm{mM}$ phenylmethanesulfonylfluoride and $2 \mathrm{mM}$ ethylenediaminetetraacetic acid (final concentration). After $5 \mathrm{~min}, 10 \mathrm{mM}$ dithiothreitol (final concentration) was added to the samples. The suspension was sonicated at $200 \mathrm{~W}$ for $15 \mathrm{~min}$ and then centrifuged at $30,000 \mathrm{~g}$ and $4^{\circ} \mathrm{C}$ for $15 \mathrm{~min}$. The supernatant was mixed well with $5 \mathrm{X}$ volume chilled acetone containing $10 \%$ trichloroacetic acid $(\mathrm{v} / \mathrm{v})$ and incubated at $-20^{\circ} \mathrm{C}$ overnight. After centrifugation at $30,000 \mathrm{~g}$ and $4^{\circ} \mathrm{C}$, the supernatant was discarded. The precipitate was washed with chilled acetone three times. The pellet was air-dried and dissolved in lysis buffer (7 M urea, $2 \mathrm{M}$ thiourea, $4 \%$ nonyl phenoxypolyethoxylethanol, $20 \mathrm{mM}$ Tris- $\mathrm{HCl}, \mathrm{pH}$ 8.0-8.5). The suspension was sonicated at 200 $\mathrm{W}$ for $15 \mathrm{~min}$ and centrifuged at $30,000 \mathrm{~g}$ and $4^{\circ} \mathrm{C}$ for $15 \mathrm{~min}$. The supernatant was transferred to another tube. To reduce disulfide bonds in proteins of the supernatant, $10 \mathrm{mM}$ dithiothreitol (final concentration) was added and incubated at $56^{\circ} \mathrm{C}$ for $1 \mathrm{~h}$. Subsequently, $55 \mathrm{mM}$ 2-iodoacetamide (final concentration) was added to block the cysteines, followed by incubation for $1 \mathrm{~h}$ in the dark. The supernatant was mixed well with $55 \mathrm{X}$ volume chilled acetone for $2 \mathrm{~h}$ at $-20^{\circ} \mathrm{C}$ to precipitate proteins. After centrifugation at $30,000 \mathrm{~g}$ and $4^{\circ} \mathrm{C}$, the supernatant was discarded and the pellet was air-dried for $5 \mathrm{~min}$, dissolved in $500 \mu \mathrm{L} 0.5 \mathrm{M}$ tetraethylammonium bromide (Applied Biosystems, Milan, Italy), and sonicated at $200 \mathrm{~W}$ for $15 \mathrm{~min}$. Finally, samples were centrifuged at $30,000 \mathrm{~g}$ and $4^{\circ} \mathrm{C}$ for $15 \mathrm{~min}$. The supernatant was transferred to a new tube and quantified using GCT. The proteins in the supernatant were kept at $-80^{\circ} \mathrm{C}$ for further analysis.

\section{iTRAQ labeling and strong cation-exchange chromatography (SCX) fractionation}

Total protein $(100 \mu \mathrm{g})$ was taken out of each sample solution and digested with Trypsin Gold (Promega, Madison, WI, USA) in the ratio protein:trypsin $=30: 1$ at $37^{\circ} \mathrm{C}$ for $16 \mathrm{~h}$. After trypsin digestion, peptides were dried by vacuum centrifugation. Peptides were reconstituted in $0.5 \mathrm{M}$ tetraethylammonium bromide and processed according to the manufacturer protocol for 8-plex iTRAQ reagent (Applied Biosystems). Briefly, one unit iTRAQ reagent was thawed and reconstituted in $24 \mu \mathrm{L}$ isopropanol. Samples were labeled with the iTRAQ tags as follows: GCT tissues sample (GCT_C tag) and normal bone tissues sample (GCT_P tag). The peptides were labeled with the isobaric tags and incubated at room temperature for $2 \mathrm{~h}$. The labeled peptide mixtures were then pooled and dried by vacuum centrifugation. SCX was performed with an LC20AB high-performance liquid chromatography (HPLC) pump system (Shimadzu, Kyoto, Japan). 
The iTRAQ-labeled peptide mixtures were reconstituted with $4 \mathrm{~mL}$ buffer A $\left(25 \mathrm{mM} \mathrm{NaH}_{2} \mathrm{PO}_{4}\right.$ in $25 \%$ acetonitrile, $\mathrm{pH} 2.7$ ) and loaded onto a $4.6 \times 250-\mathrm{mm}$ Ultremex SCX column containing 5- $\mu \mathrm{m}$ particles (Phenomenex, California, U.S.A). The peptides were eluted at a flow rate of $1 \mathrm{~mL} / \mathrm{min}$ with a gradient of buffer $A$ for $10 \mathrm{~min}, 5-60 \%$ buffer $\mathrm{B}\left(25 \mathrm{mM} \mathrm{NaH}_{2} \mathrm{PO}_{4}, 1 \mathrm{M} \mathrm{KCl}\right.$ in $25 \%$ acetonitrile, pH 2.7) for $27 \mathrm{~min}$, and $60-100 \%$ buffer $B$ for 1 min. The system was then maintained at $100 \%$ buffer $\mathrm{B}$ for $1 \mathrm{~min}$ before equilibrating with buffer $\mathrm{A}$ for $10 \mathrm{~min}$ prior to the next injection. Elution was monitored by measuring the absorbance at $214 \mathrm{~nm}$, and fractions were collected every minute. The eluted peptides were pooled into 20 fractions, desalted with a Strata X C18 column (Phenomenex), and vacuum dried.

\section{Liquid chromatography-electrospray ionization-tandem mass spectrometry (LC-ESI-MS/MS) analysis based on TripleTOF 5600}

Each fraction was resuspended in buffer A (5\% acetonitrile, $0.1 \%$ formic acid) and centrifuged at $20,000 \mathrm{~g}$ for $10 \mathrm{~min}$; the final concentration of peptide was about $0.5 \mu \mathrm{g} / \mu \mathrm{L}$ on average. Supernatant $(10 \mu \mathrm{L})$ was loaded onto a 2-cm C18 trap column of the LC-20AD NanoHPLC equipment (Shimadzu, Kyoto, Japan) using an autosampler. The peptides were then eluted onto a 10-cm analytical C18 column (inner diameter $75 \mu \mathrm{m}$ ) packed in-house. The samples were loaded at $8 \mu \mathrm{L} / \mathrm{min}$ for $4 \mathrm{~min}$, then the $35-\mathrm{min}$ gradient was run at $300 \mathrm{~nL} / \mathrm{min}$ starting from 2 to $35 \%$ B (95\% acetonitrile, $0.1 \%$ formic acid), followed by 5 min linear gradient to $60 \%, 2 \mathrm{~min}$ linear gradient to $80 \%, 80 \% \mathrm{~B}$ for $4 \mathrm{~min}$, and finally returned to $5 \%$ for $1 \mathrm{~min}$. Data acquisition was performed with a TripleTOF 5600 System (AB SCIEX, Concord, ON, Canada) fitted with a NanoSpray III source (AB SCIEX) and a pulled quartz tip as the emitter (New Objectives, Woburn, MA, USA). Data were acquired using an ion spray voltage of $2.5 \mathrm{kV}$, curtain gas of $30 \mathrm{psi}$, nebulizer gas of $15 \mathrm{psi}$, and an interface heater temperature of $150^{\circ} \mathrm{C}$. The mass spectrometer was operated with a resolving power of $\geq 30,000$ full width at half maximum for TOF MS scans. For informationdependent data acquisition, survey scans were acquired in $250 \mathrm{~ms}$ and as many as 30 product ion scans were collected if exceeding a threshold of 120 counts/s and with a 2+ to 5+ charge-state. Total cycle time was fixed to $3.3 \mathrm{~s}$. The Q2 transmission window was $100 \mathrm{Da}$ for $100 \%$. Four time bins were summed for each scan at a pulse frequency of $11 \mathrm{kHz}$ through monitoring of the $40-\mathrm{GHz}$ multichannel TDC detector with four-anode channel detect ion. A sweeping collision energy setting of $35 \pm 5 \mathrm{eV}$ coupled with iTRAQ adjust rolling collision energy was applied to all precursor ions for collision-induced dissociation. Dynamic exclusion was set for $1 / 2$ of peak width (15 s), and then the precursor was refreshed off the exclusion list.

\section{Data analysis}

Raw data files acquired from the orbitrap were converted into Mascot generic format files using Proteome Discoverer 1.2 (PD 1.2, Thermo) (5600 msconverter), and the Mascot generic format files were searched. Protein identifications were performed by using the Mascot search engine version 2.3.02 (Matrix Science, London, UK). For protein identification, a mass tolerance was permitted for intact peptide masses, with allowance for one missed cleavage in the trypsin digests. Gln->pyro-Glu (N-term Q), Oxidation (M), Deamidated (NQ) as the potential variable modifications, and Carbamidomethyl (C), iTRAQ8plex (N-term), iTRAQ8plex (K) as fixed modifications. The charge states of peptides were set to +2 and +3 . Specifically, an automatic decoy 
database search was performed in Mascot by choosing the decoy checkbox in which a random sequence of database is generated and tested for raw spectra as well as the real database. To reduce the probability of false peptide identification, only peptides with significance scores $\geq 20$ at the $99 \%$ confidence interval by a Mascot probability analysis greater than "identity" were counted as identified. Each reliable protein identification involved at least one unique peptide. For protein quantitation, it was required that a protein contained at least two unique peptides. The quantitative protein ratios were weighted and normalized by the median ratio in Mascot. We only used ratios with $P$ values $<0.05$, and only fold-changes of $>1.2$ were considered to be significant.

\section{Function method description}

Functional annotations of the proteins were conducted using the Blast2GO program against the non-redundant protein database (NR; NCBI). The Kyoto Encyclopedia of Genes and Genomes (KEGG) database (http://www.genome.jp/kegg/) and the cluster of orthologous groups of proteins (COG) database (http://www.ncbi.nlm.nih.gov/COG/) were used to classify and group the identified proteins. Gene Ontology (GO) is an international standardization of gene function classification system. It provides a dynamically updating controlled vocabulary to describe genes and gene product attributes in the organism. GO has three ontologies that can describe molecular function, cellular component, and biological process. COG is a database used for classifying orthologous proteins. The KEGG PATHWAY is a collection of manually drawn pathway maps representing our knowledge of the molecular interaction and reaction networks. Molecules are represented as nodes, and the biological relationship between two nodes is represented as an edge (line).

\section{RESULTS}

The iTRAQ analysis of proteins in the GCT tissues was performed. In the GCT, a total of $347,709 \mathrm{MS} / \mathrm{MS}$ spectra were acquired from all iTRAQ runs, among which 38,683 spectra were utilized to assign 11,340 unique peptides, representing 3101 proteins. All spectra were searched against a decoy database with all Swiss-Prot sequences reversed. Only proteins that were present at a confidence interval of $95 \%$ or greater and identified by at least two peptides were considered.

The foregoing are defined as the differential expression proteins whose difference multiples were 1.2-fold or more and whose statistical test $P$ value was less than 0.05 . A total of 467 differential expression proteins were identified in the GCT tissues, among which 311 proteins were upregulated and 156 proteins were downregulated. Among them, we selected three proteins (HSP 90-alpha, HSP beta-1, and HSP beta-2), which were expressed in the GCT tissue and were upregulated (Table 1). The three differentially expressed proteins produced $131 \mathrm{MS} / \mathrm{MS}$ spectra.

Table 1. Three proteins HS90A, HSPB1 and HSPB2 were differentially expressed.

\begin{tabular}{llcccccc}
\hline Accession & Description & Mass (Da) & Coverage (\%) & iTRAQ ratio & Sig & GO Cellular_Component & Peptide \\
\hline sp|P07900| & $\begin{array}{l}\text { HS90A_HUMAN } \\
\text { Heat-shock protein HSP 90-alpha }\end{array}$ & 109646 & 32.4 & 1.833 & $0.02^{*}$ Melanosome; cytosol; plasma membrane & 20 \\
$\begin{array}{l}\text { sp|P04792| } \\
\begin{array}{l}\text { HSPB1_HUMAN } \\
\text { Heat-shock protein beta-1 }\end{array}\end{array}$ & 25259 & 51.7 & 2.511 & $0.01^{*}$ Cytosol; cell surface; spindle; proteasome & $\begin{array}{l}\text { complex; Z disc; plasma } \\
\text { sp|Q16082| }\end{array}$ \\
$\begin{array}{l}\text { HSPB2_HUMAN } \\
\text { Heat-shock protein beta-2 }\end{array}$ & 20886 & 20.9 & 1.186 & $0.00^{*}$ Cytosol; nucleus & 3 \\
\hline
\end{tabular}

iTRAQ ratio-value $>1.0$ indicates up-regulation; iTRAQ ratio-value $<1.0$ indicate down-regulation. (the Sig is *). 
All COG proteins are assumed to derive from the same ancestral protein. Not only do "orthologs" or "paralog orthologs" come from different species of proteins that evolved from a vertical home system (speciation), but they also have the same specific function as the original protein. Paralogs are derived from genetic replication proteins in certain species and may evolve new functions that are related to the original protein function. The purpose of this analysis was to compare the identified proteins with the COG database and explore these proteins' functions or their functional classifications (Figure 1).

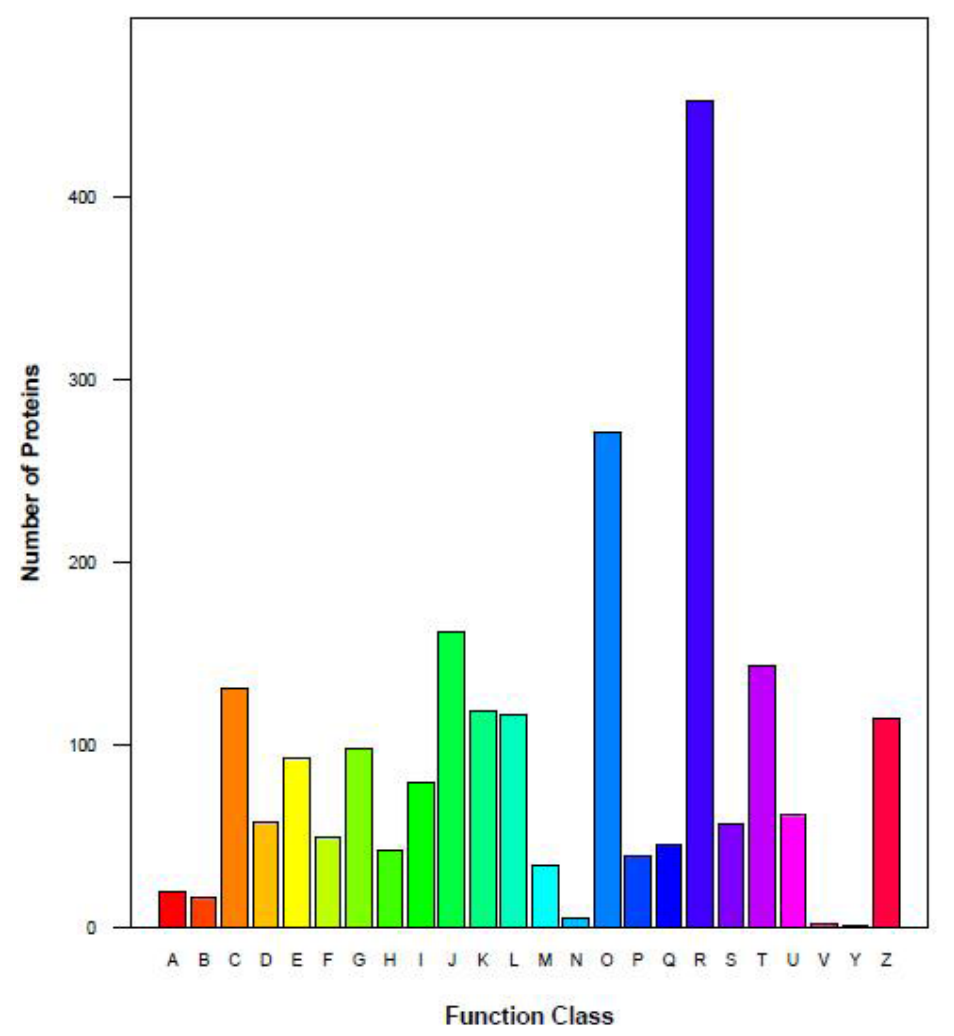

Figure 1. Statistics for the different functional proteins in the samples. Cluster of orthologous groups of proteins (COG) function classification of Homo_bone sequence (A. RNA processing and modification; B. chromatin structure and dynamics; C. energy production and conversion; D. cell cycle control, cell division, chromosome partitioning; E. amino acid transport and metabolism; F. nucleotide transport and metabolism; G. carbohydrate transport and metabolism; H. coenzyme transport and metabolism; I. lipid transport and metabolism; J. translation, ribosomal structure, and biogenesis; K. transcription; L. replication, recombination and repair; M. cell wall/membrane/envelope biogenesis; N. cell motility; O. posttranslational modification, protein turnover, chaperones; P. inorganic ion transport and metabolism; Q. secondary metabolites biosynthesis, transport and catabolism; R. general function prediction only; S. function unknown; T. signal transduction mechanisms; U. intracellular trafficking, secretion, and vesicular transport; V. defense mechanisms; Y. nuclear structure; $\mathbf{Z}$. cytoskeleton).

HSPB1 is a plasma protein and a member of the proteasome complex. It is involved in the regulation of several pathways such as the mitogen-activated protein kinase signaling pathway, Epstein-Barr virus infection, amoebiasis, and the vascular endothelial growth factor (VEGF) signaling pathway (Figure 2). 


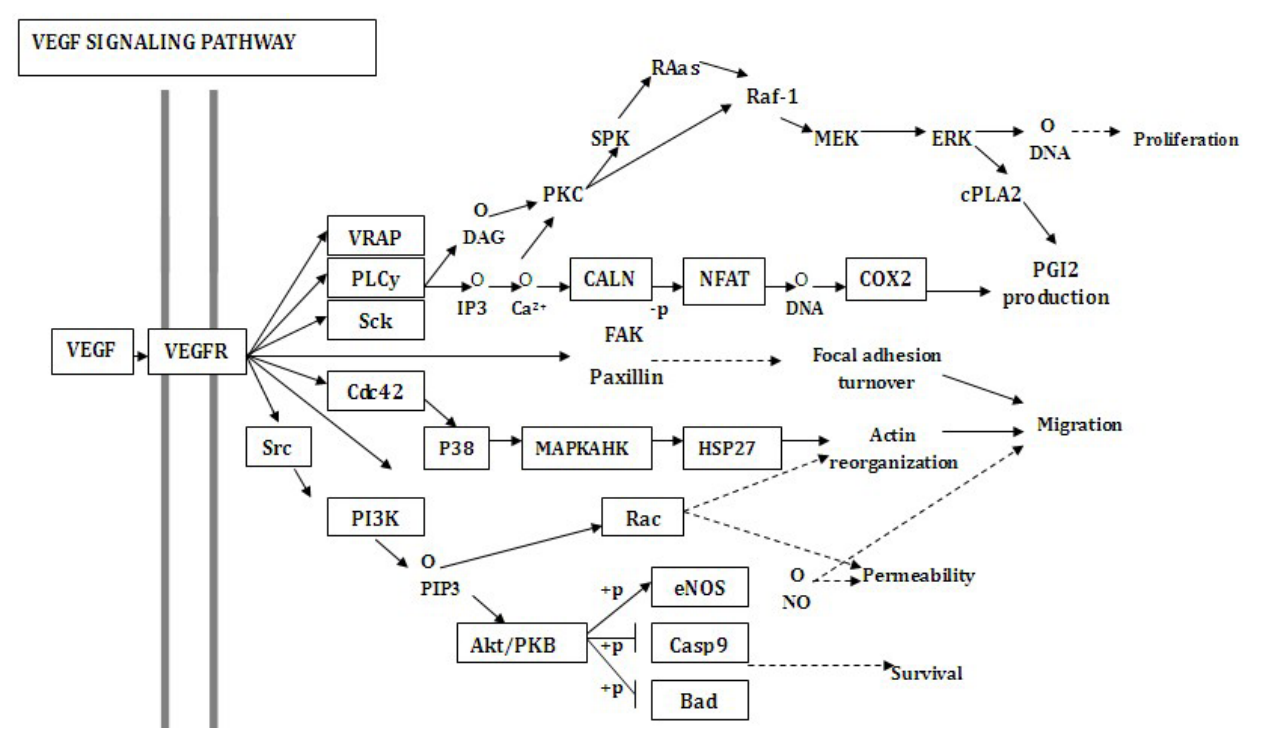

Figure 2. Pathway of vascular endothelial growth factor (VEGF) signaling.

The VEGF signaling pathway is closely related to the migration of tumors. GO analysis has shown that HSPB1 actively participates in several biological processes, including: GO:0001525// angiogenesis; GO:0009615//response to virus; GO:0035924//cellular response to VEGF stimulus; GO:0042535//positive regulation of tumor necrosis factor biosynthesis; GO:0043534//blood vessel endothelial cell migration; GO:0032731//positive regulation of interleukin-1 beta production; GO:0006446//regulation of translational initiation; GO:0035767//endothelial cell chemotaxis; GO:0071901//negative regulation of protein serine/threonine kinase activity; GO:0016071//mRNA metabolic process; GO:0006986//response to unfolded protein; and GO:0043122//regulation of the I-kappaB kinase/NF-kappaB cascade.

HS90A is a plasma membrane protein found in melanosomes. It is involved in the regulation of several pathways such as pathways in cancer, protein processing in endoplasmic reticulum, prostate cancer, antigen processing and presentation, the NOD-like receptor signaling pathway, and progesterone-mediated oocyte maturation. Pathways in cancer are closely related to the proliferation and apoptosis of tumor cells. GO analysis has shown that HS90A actively participates in several biological processes, including: GO:0007411//axon guidance; GO:0051131//chaperonemediated protein complex assembly; GO:0045040//protein import into the mitochondrial outer membrane; GO:0045429//positive regulation of nitric oxide biosynthesis; GO:0050999//regulation of nitric-oxide synthase activity; GO:0007165//signal transduction; GO:0042026//protein refolding; GO:0006200//ATP catabolic process; GO:0000086//G2/M transition of mitotic cell cycle; and GO:0006986//response to unfolded protein.

HSPB2 is a nuclear protein and is found in the cytosol. HSPB2 is involved in the regulation of several pathways such as Epstein-Barr virus infection. However, this pathway is not directly related to the biological behavior of tumor cells. GO analysis has shown that HSPB2 actively participates in several biological processes, including: GO:0043085//positive regulation of catalytic activity; and GO:0006986//response to unfolded protein. 


\section{DISCUSSION}

Numerous successful explorations of the molecular mechanisms of gene transcription in GCT have been reported. Compared with genomes and transcriptomes, which are relatively static and are transcribed into a variety of functionally distinct proteins, proteomes may provide a more realistic picture of functional aberrations. Proteomics is at the frontier of post-genomic protein study; new proteomic methods and a large number of achievements are emerging. Traditional 2-D gel electrophoresis techniques have been used extensively for comparative proteomics with significant achievements, but they present several disadvantages. ITRAQ quantitative proteomics overcomes these deficiencies and increases the number and types of differentially expressed proteins identified because it offers sensitivity, such as the ability to accurately identify membrane proteins. In contrast to 2-D gel electrophoresis, iTRAQ technology has better repeatability and quantification accuracy (Ross et al., 2004).

In this study, a stable isotope-labeled strategy using iTRAQ coupled with 2-D LC-MS/ MS was performed to separate and identify the differentially expressed proteins. A total of four HSPs (HS90A, HSPB1, HSPB2, and B4DR68) were found in the GCT tissues. HSPs are the most highly expressed cellular proteins across all species. They account for $1-2 \%$ of total proteins in unstressed cells. However, when cells are heated or subjected to other stresses, the fraction of HSPs increases to 4-6\% of all cellular proteins expressed (Lindquist and Craig, 1988).

The molecular weights of HSPs can range from 10 to $150 \mathrm{kDa}$ and they are named according to their molecular weights, for example: HSP10, HSP27, HSP60, HSP70, HSP75, HSP90 AND HSP105. The HSP structure across various species has been described in several previous studies.

A number of proteomics analyses regarding the action of HSPs in bone tumor have been conducted. New proteins have been identified that are involved in tumor cell survival and are associated with increased aggressiveness of GCTs that leads, in most cases, to local recurrences and, more rarely, to metastasis (Conti et al., 2011). PTHrP excreted by GCT stromal cells increases bone tumor cell local invasiveness and migration (Mak et al., 2013). Aberrant expression of cytoskeletaland microtubule-associated proteins in osteosarcoma may promote tumor invasion and metastasis by affecting the stability of microtubules, which consequently influences patient prognosis. Identified upregulated proteins include VIM, TUBA1C, ZNF133, EZR, ACTG1, and TF, and downregulated proteins include ADCY1, ATP5B, TUBB, RCN3, ACTB, and YWHAZ (Li et al., 2010).

HSPB1 (HSP27) is expressed specifically on cell surfaces, in the cytosol, spindles, proteasome complexes, $Z$ discs, and in the plasma. It is related to the occurrence of tumors, and is involved in biological functions such as cellular response to vascular endothelial growth factor stimulus, positive regulation of tumor necrosis factor biosynthesis, and blood vessel endothelial cell migration. HSPB1 is highly expressed in the tumor tissues of patients diagnosed with cancer, and expression levels correlate with poor prognosis. HSPB1 plays a dual role in cancer and promotes cancer development by suppressing host anti-cancer response, such as apoptosis and senescence, and facilitates the enhanced expression of metastatic genes (Nagaraja et al., 2012). HSPB1mediated protection from tumor cell apoptosis induced by chemotherapeutic drugs occurs through several mechanisms, including decreased production of reactive oxygen species, restoration of protein homeostasis and promotion of cell survival by protein folding, stabilization of the actincytoskeleton, delayed release of cytochrome c from mitochondria, and inhibition of caspase-3 activation (Li et al., 2010). Developing HSPB1 structure-based interfering strategies could lead to 
the discovery of new anti-cancer drugs (Arrigo and Gibert, 2012). HSPB1 and HSP90AA1 might be novel tumor antigens for immunotherapy of myeloma (Li et al., 2014). FABP5, HSPB1, and MnSOD may be potential biomarkers for pelvic lymph node metastasis of cervical cancer, and I-Ilst may have an important role in the pathogenesis of pelvic lymph node metastasis (Wang et al., 2014a). Several client proteins with potential pro-cancerous roles have so far been found to interact with HSPB1 and its closely related family members HSPB5 and HSPB4 (Arrigo and Gibert, 2014). Soluble heat-shock protein B1 (HSPB1) is released primarily from endothelial cells and regulates angiogenesis via direct interaction with VEGF. Matrix metalloproteinase inhibitors may have pro-tumorigenic effects by reducing HSPB1 fragmentation (Choi et al., 2014). HSPB1 is a promising diagnostic marker for clear cell renal cell carcinoma, although further large-scale studies are required (White et al., 2014). A novel mechanism by which HSPB1 recognizes Nemolike kinase in breast cancer cells and prevents Nemo-like kinase-mediated cell apoptosis has been described (Shaw-Hallgren et al., 2014). A large number of researchers have paid close attention to HSPB1 inhibitor in the context of renal carcinoma, breast cancer, cervical cancer, etc. However, the role of HSPB1 in GCT has not been reported. Hopefully our study will be helpful to further research. HS90A, a member of the HSP90 family, is expressed specifically in the melanosomes, cytosol, and plasma membrane. It is involved in biological functions such as protein import into the mitochondrial outer membrane, signal transduction, protein refolding, transition of the mitotic cell cycle, and the response to unfolded protein. These functions are related to tumor formation. Positive selection and signatures of functional divergence after duplication are reported to be facilitators of rapid evolutionary change, and are targets for anticancer therapeutics (Carretero-Paulet et al., 2013). Vorinostat increases acetylation of HSP90 and alpha tubulin, and decreases HSP90 client protein and HDAC6 expression in human breast cancers in vivo (Tu et al., 2014). HSP90a, p53, MDM2, Bcl-2, Bax, cytochrome c, and cleaved caspase-3 might be involved in apoptosis regulation and be associated with clinical outcomes of ALK+ systemic anaplastic large-cell lymphoma (Li et al., 2011). P53 acetylation status and the levels of HSP90 may participate in modulation of nutlin-3 sensitivity in acute myeloid leukemia (Haaland et al., 2014). A novel mechanism revealed for human carcinogenesis via methylation of HSP90AB1 by SMYD2, and additional functional studies may assist in developing novel strategies for cancer therapy (Hamamoto et al., 2014). SNX-25a, a novel HSP90 inhibitor, inhibits human cancer growth more potently than 17-AAG (Wang et al., 2014b). Inhibition of HSP90 downregulates both HIF-1 $\alpha$ and NF-KB, leading to inhibition of epithelialmesenchymal transition, motility, and invasiveness in colorectal cancer (Nagaraju et al., 2014). The specific HSP90 inhibitor AUY922 plays a therapeutic role in the treatment of adrenocortical carcinoma and, therefore, HSP90 could qualify as a promising new target in adrenocortical carcinoma (Huang et al., 2014). Taking into account the role of HS90A in tumor progression, and the involvement of Akt kinase in cell signaling and the anti-apoptotic pathways in tumors, chemical and molecular double target treatment offers a novel therapeutic strategy (Mehta et al., 2011). In recent years, most researchers have concentrated on HSP90 inhibitor for breast cancer, acute myeloid leukemia, colorectal cancer, etc. HSP90 inhibitor for GCT has not been reported, and we did not search the reports concerning the role of HSPB1 in GCT. Our study may be helpful in researching the inhibitor for GCT.

HSPB2 is expressed specifically in the cytosol and nucleus, and is involved in biological functions such as positive regulation of catalytic activity, and response to unfolded protein. These functions have a close relationship with tumor formation. Both HSPB2 and aB-crystallin are linked with human renal carcinogenesis. These findings provide novel insights into the role of p53 as a 
regulator of the bidirectional gene pair HSPB2/aB-crystallin-mediated reactive oxygen species and the Warburg effect (Liu et al., 2014). HSPB2/myotonic dystrophy protein kinase binding protein as a novel molecular chaperone exhibits target protein-dependent chaperone-like activity (Prabhu et al., 2012). Reports on the relationship between HSPB2 and tumors are scarce, especially those concerning HSPB2 and GCT.

ITRAQ analysis of GCT tissues is an effective method for proteomic investigation. The aim of this study was to identify differentially expressed proteins that can be used to monitor recurrence and metastasis of GCT under the assumption that morphologic changes are accompanied by the abnormal expression of proteins. Our findings indicate that HSPs are important predictors of the aggressive behavior of GCT. However, additional research using a larger number of GCT patients is needed to confirm our findings. Future studies will be conducted to explore HSP inhibitor to prevent tumor recurrence and migration.

\section{Conflicts of interest}

The authors declare no conflict of interest.

\section{ACKNOWLEDGMENTS}

We gratefully acknowledge the National Natural Science Foundation of China, which is our sponsoring organization.

\section{REFERENCES}

Arrigo AP and Gibert B (2012). HspB1 dynamic phospho-oligomeric structure dependent interactome as cancer therapeutic target. Curr. Mol. Med. 12: 1151-1163.

Arrigo AP and Gibert B (2014). HSPB1, HspB5 and HspB4 in Human Cancers: Potent Oncogenic Role of Some of Their Client Proteins. Cancers 6: 333-365.

Badowska-Kozakiewicz AM and Malicka E (2012). Immunohistochemical evaluation of expression of heat shock proteins HSP70 and HSP90 in mammary gland neoplasms in bitches. Pol. J. Vet. Sci. 15: 209-214.

Campanacci M (1993). Giant cell tumor of bone. Curr. Orthop. 7: 26-35.

Carretero-Paulet L, Albert VA and Fares MA (2013). Molecular evolutionary mechanisms driving functional diversification of the HSP90A family of heat shock proteins in eukaryotes. Mol. Biol. Evol. 30: 2035-2043.

Choi SH, Lee HJ, Jin YB, Jang J, et al. (2014). MMP9 processing of HSPB1 regulates tumor progression. PLoS One 9: e85509.

Chow SN, Chen RJ, Chen CH, Chang TC, et al. (2010). Analysis of protein profiles in human epithelial ovarian cancer tissues by proteomic technology. Eur. J. Gynaecol. Oncol. 31: 55-62.

Cohen M, Dromard M and Petignat P (2010). Heat shock proteins in ovarian cancer: a potential target for therapy. Gynecol. Oncol. 119: 164-166.

Conti A, Rodriguez GC, Chiechi A, Blazquez RM, et al. (2011). Identification of potential biomarkers for giant cell tumor of bone using comparative proteomics analysis. Am. J. Pathol. 178: 88-97.

Ernoult E, Gamelin E and Guette C (2008). Improved proteome coverage by using iTRAQ labelling and peptide OFFGEL fractionation. Proteome Sci. 6: 27.

Gamberi G, Benassi MS, Böhling T, Ragazzini P, et al. (1998). Prognostic relevance of C-myc gene expression in giant-cell tumor of bone. J. Orthop. Res. 16: 1-7.

Gupta R, Seethalakshmi V, Jambhekar NA, Prabhudesai S, et al. (2008). Clinicopathologic profile of 470 giant cell tumors of bone from a cancer hospital in western India. Ann. Diagn. Pathol. 12: 239-248.

Haaland I, Opsahl JA, Berven FS, Reikvam H, et al. (2014). Molecular mechanisms of nutlin-3 involve acetylation of p53, histones and heat shock proteins in acute myeloid leukemia. Mol. Cancer 13: 116.

Hamamoto R, Toyokawa G, Nakakido M, Ueda K, et al. (2014). SMYD2-dependent HSP90 methylation promotes cancer cell proliferation by regulating the chaperone complex formation. Cancer Lett. 351: 126-133. 
Huang J, Sun C, Zhang T, Pan L, et al. (2014). Potent antitumor activity of HSP90 inhibitor AUY922 in adrenocortical carcinoma. Tumour Biol. 35: 8193-8199.

Li HL, Huang XP, Zhou XH, Ji TH, et al. (2011). Correlation of seven biological factors (Hsp90a, p53, MDM2, Bcl-2, Bax, Cytochrome $\mathrm{C}$, and Cleaved caspase3) with clinical outcomes of ALK+ anaplastic large-cell lymphoma. Biomed. Environ. Sci. 24: 630-641.

Li R, Qian J, Zhang W, Fu W, et al. (2014). Human heat shock protein-specific cytotoxic T lymphocytes display potent antitumour immunity in multiple myeloma. Br. J. Haematol. 166: 690-701.

Li Y, Liang Q, Wen YQ, Chen LL, et al. (2010). Comparative proteomics analysis of human osteosarcomas and benign tumor of bone. Cancer Genet. Cytogenet. 198: 97-106.

Lindquist S and Craig EA (1988). The heat-shock proteins. Annu. Rev. Genet. 22: 631-677.

Liu S, Yan B, Lai W, Chen L, et al. (2014). As a novel p53 direct target, bidirectional gene HspB2/aB-crystallin regulates the ROS level and Warburg effect. Biochim. Biophys. Acta 1839: 592-603.

Mak IW, Turcotte RE and Ghert M (2013). Parathyroid hormone-related protein (PTHrP) modulates adhesion, migration and invasion in bone tumor cells. Bone 55: 198-207.

Mehta A, Shervington L, Munje C and Shervington A (2011). A novel therapeutic strategy for the treatment of glioma, combining chemical and molecular targeting of hsp90a. Cancers 3: 4228-4244.

Molloy MP (2000). Two-dimensional electrophoresis of membrane proteins using immobilized pH gradients. Anal. Biochem. 280: $1-10$.

Nagaraja GM, Kaur P and Asea A (2012). Role of human and mouse HspB1 in metastasis. Curr. Mol. Med. 12: 1142-1150.

Nagaraju GP, Long TE, Park W, Landry JC, et al. (2014). Heat shock protein 90 promotes epithelial to mesenchymal transition, invasion, and migration in colorectal cancer. Mol. Carcinog 54: 1147-1158.

Niknejad H, Yazdanpanah G, Mirmasoumi M, Abolghasemi H, et al. (2013). Inhibition of HSP90 could be possible mechanism for anti-cancer property of amniotic membrane. Med. Hypotheses. 81: 862-865.

Prabhu S, Raman B, Ramakrishna T, Rao ChM (2012). HspB2/myotonic dystrophy protein kinase binding protein (MKBP) as a novel molecular chaperone: structural and functional aspects. PLoS One 7: e29810.

Rabilloud T (2009). Membrane proteins and proteomics: love is possible, but so difficult. Electrophoresis 30: S174-180.

Ross PL, Huang YN, Marchese JN, Williamson B, et al. (2004). Multiplexed protein quantitation in Saccharomyces cerevisiae using amine-reactive isobaric tagging reagents. Mol. Cell. Proteomics 3: 1154-1169.

Shadforth IP, Dunkley TP, Lilley KS and Bessant C (2005). i-Tracker: for quantitative proteomics using iTRAQ. BMC Genomics 6: 145.

Shaw-Hallgren G, Chmielarska Masoumi K, Zarrizi R, Hellman U, et al. (2014). Association of nuclear-localized Nemo-like kinase with heat-shock protein 27 inhibits apoptosis in human breast cancer cells. PLoS One 9: e96506.

Tu Y, Hershman DL, Bhalla K, Fiskus W, et al. (2014). A phase I-II study of the histone deacetylase inhibitor vorinostat plus sequential weekly paclitaxel and doxorubicin-cyclophosphamide in locally advanced breast cancer. Breast Cancer Res. Treat. 146: 145-152.

Wang S, Wang X, Du Z, Liu Y, et al. (2014a). SNX-25a, a novel Hsp90 inhibitor, inhibited human cancer growth more potently than 17-AAG. Biochem. Biophys. Res. Commun. 450: 73-80.

Wang W, Jia HL, Huang JM, Liang YC, et al. (2014b). Identification of biomarkers for lymph node metastasis in early-stage cervical cancer by tissue-based proteomics. Br. J. Cancer 110: 1748-1758.

Werner M (2006). Giant cell tumour of bone: morphological, biological and histogenetical aspects. Int. Orthop. 30: 484-489.

White NM, Masui O, Desouza LV, Krakovska O, et al. (2014). Quantitative proteomic analysis reveals potential diagnostic markers and pathways involved in pathogenesis of renal cell carcinoma. Oncotarget 5: 506-518.

Wu WW, Wang G, Baek SJ and Shen RF (2006). Comparative study of three proteomic quantitative methods, DIGE, cICAT, and ITRAQ, using 2D gel- or LC-MALDI TOF/TOF. J. Proteome Res. 5: 651-658.

Yan W and Chen SS (2005). Mass spectrometry-based quantitative proteomic profiling. Brief Funct. Genomic Proteomic. 4: 27-38.

Zhang S, Mercado-Uribe I, Hanash S and Liu J (2013). iTRAQ-based proteomic analysis of polyploid giant cancer cells and budding progeny cells reveals several distinct pathways for ovarian cancer development. PLoS One 8: e80120.

Zhao M, Ding JX, Zeng K, Zhao J, et al. (2014). Heat shock protein 27: a potential biomarker of peritoneal metastasis in epithelial ovarian cancer? Tumour Biol. 35: 1051-1056. 